\title{
In vitro maturation of immature oocytes for fertility preservation in cancer patients compared to control patients with fertility problems in an in vitro fertilization program
}

\author{
Irma Virant-Klun'1, Jure Bedenk², Nina Jancar² \\ ${ }^{1}$ Clinical Research Centre, University Medical Centre Ljubljana, Ljubljana, Slovenia \\ ${ }^{2}$ Division of Obstetrics and Gynecology, University Medical Centre Ljubljana, Ljubljana, Slovenia
}

Radiol Oncol 2022; 56(1): 119-128.

Received 5 August 2021

Accepted 25 November 2021

Correspondence to: Prof. Irma Virant-Klun, Ph.D., Clinical Research Centre, University Medical Centre Ljubljana, Zaloška cesta 2, SI 1000 Ljubljana, Slovenia. E-mail: irma.virant@kclj.si

Disclosure: No potential conflicts of interest were disclosed.

This is an open access article under the CC BY-NC-ND license (http://creativecommons.org/licenses/by-nc-nd/4.0/).

\begin{abstract}
Background. The aim of this study was to determine whether in vitro maturation (IVM) of immature oocytes after controlled hormonal stimulation of the ovaries could be important in cancer patients to improve their chances of conception in the future.

Patients and methods. After ovarian stimulation in cancer patients, the number of oocytes and their quality and maturity were compared to control patients with fertility problems in the in vitro fertilization (IVF) program. In both groups of patients, immature oocytes at the developmental stage of germinal vesicle were matured in vitro and the proportion of oocytes that matured in vitro was compared between groups. In a subset of women with fertility problems, intracytoplasmic sperm injection (ICSI) was performed on IVM oocytes to assess their ability to be fertilized and develop into an embryo compared to vivo matured oocytes in the same cycles and consider the procedure in cancer patients.

Results. In patients with different cancers, the disease did not affect the number and quality of retrieved oocytes. In cancer patients, there was even a significantly lower proportion of immature oocytes than in patients with fertility problems (30.0\% vs. 43.6\%; P 0.05). However, in patients with cancer, fewer oocytes per patient matured in vitro than in patients with fertility problems ( $1.39 \pm 1.04$ vs. $2.48 \pm 1.83$; $P<0.05)$. After ICSI, the proportions of fertilized oocytes and fertilized oocytes developing into an embryo did not differ between oocytes matured in vitro and in vivo in the same cycles.

Conclusions. Oocyte IVM is proving to be a reliable procedure for resolving immature oocytes after controlled ovarian stimulation in cancer patients.
\end{abstract}

Key words: cancer; fertility preservation; oocyte; in vitro maturation; vitrification

\section{Introduction}

Many young women in the reproductive period of life who do not yet have children or would like to have another child suffer from cancer. Today, cancer therapies are successful, but unfortunately, they can negatively affect the ovarian function (in- cluding oocyte quality) and fertility. At a median of 5.0 years from initial breast cancer diagnosis, $49 \%$ patients after adjuvant chemotherapy with anthracyclines and taxanes and 11\% after therapy with tamoxifen had become post- and peri-menopausal. ${ }^{1}$ Decreased ovarian follicle reserve occurs in more than one-third of patients after breast can- 
cer treatment resulting in permanent infertility. ${ }^{2}$ In long-term female survivors of pediatric hematologic malignancies $26.7 \%$ experienced premature ovarian insufficiency and face infertility after cancer treatment. ${ }^{3}$ The situation is similar with other cancers; cancer therapy is the cause of premature ovarian failure in $25 \%$ of women with this diagnosis. ${ }^{4}$ Therefore, it is very important to consider the preservation of female fertility before oncotherapy. An improvement in the survival rates of cancer patients and recent advances in assisted reproductive technologies have led to significant progress in fertility preservation treatments.

One option is vitrification and long-term storage of the patient's oocytes for later in vitro fertilization (IVF) with partner's sperm and transfer of embryos into the uterus. This program is established in many health care institutions around the world for a variety of cancers, including breast cancer. ${ }^{5-12}$ Oocyte cryopreservation is an effective approach ${ }^{13-15}$, but it is still thought that further studies are needed in cancer patients to ensure the excellent outcomes obtained in women without cancer. ${ }^{16}$ After controlled hormonal stimulation of the ovaries, in vitro maturation (IVM) of immature oocytes before vitrification is recommended and not after vitrification/ devitrification procedure. ${ }^{17}$ Even if there are no differences in survival rates between oocytes vitrified before or after IVM procedure, decreased maturation rates of immature oocytes vitrified before IVM may be explained by underlying ultrastructural and biomolecular alterations. ${ }^{17}$

Human oocyte cryopreservation may offer some advantages compared to embryo freezing in cancer patients and also eliminates some ethical, legal, and moral concerns of embryo freezing ${ }^{17}$, and is an option in young cancer patients who are single..$^{9,18}$ However, the chance of success depends primarily on the number of oocytes that have been vitrified in the patient ${ }^{15}$ and some breast cancer patients may have contraindications to exogenous gonadotropin administration for controlled ovarian stimulation. ${ }^{19}$ Some recent data show that ovarian stimulation for oocyte vitrification does not modify disease-free survival and overall survival rates in patients with early breast cancer ${ }^{20}$ and the safety of pregnancy after an established diagnosis of breast cancer has been confirmed in numerous studies. ${ }^{21}$

In the case of vitrification and storage of oocytes, controlled hormonal stimulation of the ovaries is required to obtain oocytes. Despite careful hormonal stimulation of the ovaries, the significant proportion of oocytes obtained by ultrasoundguided aspiration of ovarian follicles is immature as metaphase I (MI) oocytes or prophase I oocytes with germinal vesicle (GV). Immature MI oocytes mostly mature spontaneously in vitro and are vitrified, while immature GV oocytes do not mature spontaneously and are incapable of fertilization. Therefore, GV oocytes are not vitrified and stored in liquid nitrogen in clinical practice and are discarded and lost to the patient. The important question is whether the maturation of these oocytes in vitro makes sense. There is a lack of data regarding the outcome of in vitro matured oocytes cryopreserved in cancer patients. ${ }^{22}$ Recently, the first birth achieved after fertility preservation using vitrification of in vitro matured oocytes in a patient with breast cancer has been reported. ${ }^{23}$

The purpose of this study was to investigate the effectiveness of maturation of immature GV oocytes of cancer patients in laboratory conditions (in maturation medium and co-culture with $\mathrm{cu}-$ mulus cells from mature oocytes of the same patients) compared to control women involved in the IVF program due to fertility problems. Because all oocytes of cancer patients are still frozen, we tried to elucidate the success of IVF procedure, actually intracytoplasmic sperm injection (ICSI), on the in vitro matured oocytes of patients with fertility problems as a model for cancer patients.

\section{Patients and methods}

This research was approved by the Slovenian National Medical Ethical Committee (No. 0120222/2016-2; KME 115/04/16). In this prospective research the immature (germinal vesicle-GV, prophase I) oocytes of two groups of patients were included: i) 45 oocytes of 18 cancer patients with predominating breast cancer (Figure 1) and ii) 74 oocytes of 21 healthy (non-cancer) patients (control) with fertility problems (partners of infertile men with impaired semen quality: oligozoospermia with less than 15 million spermatozoa/ml or teratozoospermia with less than $4 \%$ morphologically normal spermatozoa according to the World Health Organization (WHO) Criteria $2010^{24}$ who were included in the IVF program. All patients were in the reproductive period of life, aged 18 to 43 years.

\section{Oocytes after controlled hormonal stimulation of the ovaries}

In both groups of patients, both immature and mature oocytes were together retrieved after controlled hormonal stimulation of the ovaries using 
the same, antagonist protocol and ultrasoundguided aspiration of ovarian follicles. In patients with fertility problems, the stimulation was started on day 2 of the menstrual cycle with 150 to 300 I.U. of recombinant follicle-stimulating hormone (rFSH) daily. In cancer patients, the stimulation was initiated immediately after they have been sent to our department, no matter of the cycle phase. The ovarian stimulation was started with 225 to 300 I.U. of rFSH. In breast cancer patients, an aromatase inhibitor - letrozole $(2.5 \mathrm{mg}$ every 12 hours) was added to prevent estradiol rise and its possible detrimental effect on breast cancer. In all patients, the gonadotropin-releasing hormone $(\mathrm{GnRH})$ antagonist was added, when dominant follicle measured $14 \mathrm{~mm}$ in a diameter. In patients with fertility problems, the oocyte maturation was triggered with choriogonadotropin alfa-Ovitrelle, when follicles measured $18 \mathrm{~mm}$ or more. If there were more than 15 follicles in both ovaries, the maturation triggering was performed with $\mathrm{GnRH}$ agonist. In majority of cancer patients, GnRH agonist was used for oocyte maturation to prevent ovarian hyperstimulation (ovarian hyperstimulation syndrome; OHSS), but some of them, if there were less than 10 follicles in both ovaries, were also treated by Ovitrelle. All follicles with a diameter of $16 \mathrm{~mm}$ or more were aspirated in all patients. A constant aspiration pressure of $180 \mathrm{~mm} \mathrm{Hg}$ was used to aspirate the oocytes from the follicles.

Mature oocytes with expressed polar body were immediately vitrified by soaking in a mixture of cryoprotectants, direct plunging into liquid nitrogen $\left(-196^{\circ} \mathrm{C}\right)$, and stored in it, as described elsewhere. ${ }^{25}$

\section{In vitro maturation of immature oocytes}

Immature GV oocytes were exposed to the procedure of IVM in a seria of media of the IVM Maturation System (MediCult IVM ${ }^{\circledR}$ System, Origio/CooperSurgical, Denmark).

For IVM, each GV oocyte was first exposed for two hours in the LAG Medium for conditioning and then for 24 to 28 hours to the maturation medium of this system containing the reproductive hormones: follicle stimulating hormone (FSH; $75 \mathrm{mIU} /$ $\mathrm{ml}$ ) and human chorionic gonadotropin (HCG; 100 $\mathrm{mIU} / \mathrm{ml}$ ) and in a co-culture with cumulus cells denuded from mature oocytes of the same patient, as described elsewhere (Figure 2). ${ }^{26}$ During incubation in these media, oocytes were cultured in a $\mathrm{CO}_{2}{ }^{-}$ incubator at $37^{\circ} \mathrm{C}$ and $6 \% \mathrm{CO}_{2}$ in air. Oocytes were supposed to be mature (in a metaphase II) when

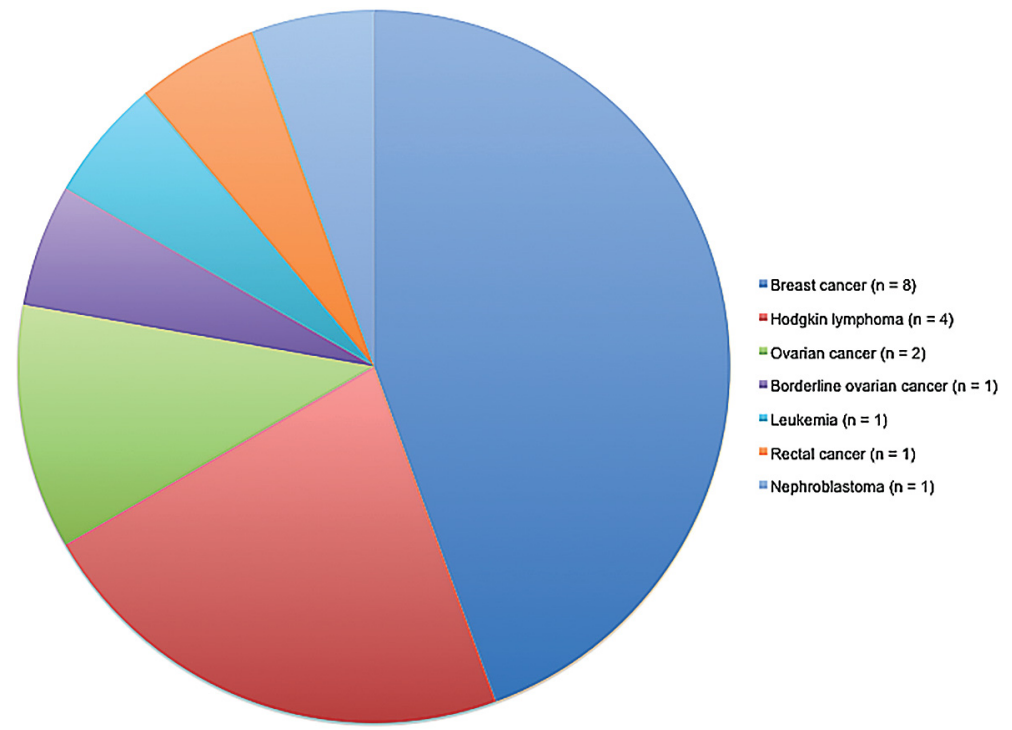

FIGURE 1. Types of disease in cancer patients included in this study. Breast cancer patients predominated.
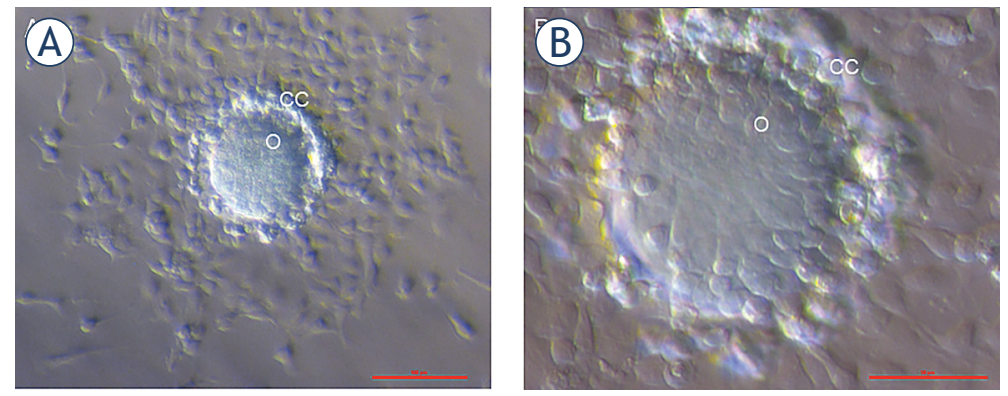

FIGURE 2. In vitro maturation of the oocyte in the maturation medium and in coculture with the cumulus cells of mature oocytes in the same patient at magnification $40 \times(\mathrm{A})$ and magnification $100 \times(\mathrm{B})$.

$\mathrm{CC}=$ cumulus (granulosa) cells; $\mathrm{O}=$ oocyte; Red bar = A) $100 \mu \mathrm{m}$ in B) $50 \mu \mathrm{m}$

they extruded a polar body. All oocytes of cancer patients and the majority of oocytes of women with fertility problems that matured in vitro were vitrified and stored in liquid nitrogen for later clinical use (IVF). Patients with $\geq 31 \%$ GV oocytes had increased oocyte immaturity. A subset of in vitro matured oocytes of women with fertility problems was fertilized in vitro. 


\section{In vitro fertilization by ICSI}

In a subgroup of 17 in vitro matured oocytes from 17 patients ( 1 oocyte per patient) with fertility problems (female partners of infertile men with oligozoospermia or teratozospermia) ICSI was performed with partner's sperm one day later after oocyte and sperm retrieval from a couple. Oocytes were denuded by hyaluronidase to remove the cumulus cells and microinjection of one spermatozoon per oocyte was performed, as described elsewhere. ${ }^{27}$ Only motile spermatozoa were used for ICSI. The next day, fertilization (presence of two pronuclei and extruded second polar body) was checked and embryo cleavage one day later. Good quality embryos were vitrified and stored in liquid nitrogen $\left(-196^{\circ} \mathrm{C}\right)$ for later clinical use (transfer to the uterus). The rates of fertilization and embryo cleavage were compared between oocytes that matured in vitro and in vivo (in the ovaries; aspirated as mature) in the same patients after controlled ovarian hormonal stimulation.

\section{Statistics}

Both groups of patients, cancer and infertile patients, were compared in terms of the number of oocytes obtained after controlled hormonal stimulation of their ovaries, the proportion of immature and degenerated oocytes, and the proportion of immature (GV) oocytes that matured in vitro. Due to the relatively small number of patients/oocytes included and the abnormal distribution of data, tested by Shapiro-Wilk normality test, non-parametric tests (Fisher's exact and Mann-Whitney U tests) were performed; statistical significance was set at $\mathrm{P}<0.05$. After ICSI, the fertilization and embryo cleavage rates of oocytes that matured in vitro were compared with oocytes of the same patients that matured in vivo and were aspirated from the ovaries as mature oocytes in the same cycle of controlled ovarian hormonal stimulation using Fisher's exact and Wilcoxon tests; statistical significance was set at $\mathrm{P}<0.05$.

\section{Results}

Cancer patients had different types of cancer, but breast cancer was predominant (Figure 1). The average age of cancer patients was $30.3 \pm 6.3$ years and of women with fertility problems $33.4 \pm 5.0$ years. The two groups of women did not differ significantly in their age. There was also no significant difference in the age of patients with breast cancer and patients with other cancers (32.0 \pm 6.2 vs. 29.0 \pm 6.0 years).

\section{Numbers, quality and immaturity of oocytes after controlled hormonal stimulation of the ovaries}

After controlled hormonal stimulation of the ovaries, 198 oocytes were retrieved in cancer patients and 259 oocytes in infertile women. Cancer patients and patients with fertility problems did not differ significantly in the number of retrieved oocytes $(11.0 \pm 9.0$ oocytes/patient vs. $12.3 \pm 9.2$ oocytes/patient), as revealed by Mann-Whitney U test. In cancer patients, the proportion of immature GV and MI oocytes was significantly lower than in patients with fertility problems $(30.0 \%$ vs. $43.6 \%$; P $<0.05$ ), as revealed by Fisher's exact test (Table 1). The groups did not differ significantly in the proportion of GV oocytes ( $23.0 \%$ vs. $28.6 \%$ ) (Table 1 ); among patients with immature oocytes, $50 \%$ of cancer patients and $48.0 \%$ of patients with fertility problems had increased proportion $(\geq 31 \%)$ of GV oocytes with a germinal vesicle, which did not differ significantly. There was also no difference in the proportion of degenerated oocytes between cancer patients and patients with fertility problems (8.6 vs. $6.5 \%$ ) (Table 1).

If we considered the type of cancer, we found that there was no significant difference in the number of all immature (MI and GV) oocytes in patients with breast cancer compared to patients with fertility problems or patients with other cancers $(4.17 \pm 3.25$ vs. $5.29 \pm 3.76$ and $2.92 \pm 2.47 \mathrm{im}-$ mature oocytes/patient, respectively), as revealed by Mann-Whitney $U$ test. There was also no statistically significant difference in the proportion of all immature (GV and MI) oocytes in breast cancer patients compared to patients with other cancers or patients with fertility problems $(36.0 \%$ vs. $27.0 \%$ and $43.6 \%$ ), as found using the Fisher's exact test. Nevertheless, there was a tendency for a significantly higher proportion of immature, GV oocytes in breast cancer patients compared to patients with other cancers $(31.43 \%$ vs. $18.75 \%$; $\mathrm{P}=0.0531$, Fisher's exact test).

\section{In vitro matured oocytes}

Forty-five GV oocytes in cancer patients and 74 GV oocytes in patients with fertility problems underwent IVM procedure (Table 2); the proportion of oocytes $(15.5 \%$ in cancer patients and $12.2 \%$ in 
women with fertility problems) degenerated just before or during conditioning in LAG medium. We found that a lower proportion of oocytes matured in vitro in cancer patients compared to patients with fertility problems (66.0 vs. $80.0 \%$ ), however, the difference was not statistically significant, as revealed by Fisher's exact test. In spite of that, number of oocytes that matured in vitro per patient was significantly lower in cancer patients than in patients with fertility problems $(1.39 \pm 1.04$ vs. 2.48 \pm 1.83 oocytes/patient; $\mathrm{P}<0.05$, Mann-Whitney $\mathrm{U}$ test), as shown in Table 2.

In cancer patients, there was also a lower proportion of oocytes that matured in vitro in patients with breast cancer than in patients with other cancers and patients with fertility problems $(54.5 \%$ vs. $81.2 \%$ and $80.0 \%$ ) (Table 2). The difference between patients with breast cancer and women with fertility problems tended to be statistically significant $(\mathrm{P}=0.0862$; Fisher's exact test $)$ and was probably not significant due to the relatively low number of oocytes included.

Overall, 198 oocytes were retrieved in cancer patients, of which 139 were mature. Following IVM, the number of total mature oocytes increased to 164 (13.0\% increase in mature oocyte yield). In patients with fertility problems, 259 oocytes were retrieved, of which 146 were mature. After IVM, the number of total mature oocytes increased to 198, which means $20.1 \%$ increase in mature oocyte yield. Thus, there was no significant difference in the yield of mature oocytes after IVM between cancer patients and patients with fertility problems. 15.5\% (7/45) GV oocytes in cancer patients and 12.2\% (9/74) GV oocytes in patients with fertility problems degenerated before in vitro maturation procedure.

\section{Results of ICSI of in vitro matured oocytes in patients with fertility problems}

In vitro fertilization of 49 oocytes in 17 patients with fertility problems (average age $34.3 \pm 4.4$ years) was performed by ICSI with partner's semen (in 2 men oligozoospermia and 15 men teratozoospermia). After performing this method, 27 (55.1\%) oocytes were fertilized (expressing two pronuclei and two polar bodies) and $23(85.2 \%)$ fertilized oocytes (zygotes) further developed into an embryo, as shown in Table 3; four zygotes did not cleave further. Good quality embryos were vitrified and stored in liquid nitrogen for future clinical use in patients (transfer into the uterus).
TABLE 1. Differences in the number, quality and immaturity of oocytes after controlled hormonal stimulation of the ovaries in cancer patients compared to patients with fertility problems

\begin{tabular}{lcc}
\hline & $\begin{array}{c}\text { Cancer } \\
\text { patients } \\
(\mathbf{n}=\mathbf{1 8})\end{array}$ & $\begin{array}{c}\text { Patients with } \\
\text { fertility problems } \\
(\mathbf{n}=\mathbf{2 1})\end{array}$ \\
\hline Age (years) & $30.3 \pm 6.3$ & $33.4 \pm 5.0$ \\
\hline Number of retrieved oocytes & 198 & 259 \\
\hline Oocytes per patient & $11.0 \pm 9.0$ & $12.3 \pm 9.2$ \\
\hline Number of degenerated oocytes & $17(8.6 \%)$ & $17(6.5 \%)$ \\
\hline Number of immature (Ml + GV) oocytes & $59(30.0 \%)^{*}$ & $113(43.6 \%)$ \\
\hline Number of immature GV oocytes & $45(23.0 \%)$ & $74(28.6 \%)$ \\
\hline
\end{tabular}

* = statistically significant difference $(P=0.0064)$ revealed by Fisher's exact test; significance was set at $\mathrm{P}<0.05 ; \mathrm{GV}=$ germinal vesicle; $\mathrm{Ml}=$ metaphase I (oocyte meiosis)

TABLE 2. Numbers and percentages of oocytes that matured in vitro in patients with different cancers compared to patients with fertility problems

\begin{tabular}{|c|c|c|}
\hline & $\begin{array}{l}\text { Number of oocytes } \\
\text { that underwent in } \\
\text { vitro maturation }\end{array}$ & $\begin{array}{l}\text { Number of oocytes that } \\
\text { matured in vitro }\end{array}$ \\
\hline $\begin{array}{l}\text { All cancer patients } \\
\qquad(n=18)\end{array}$ & $38 / 45$ & $\begin{array}{c}25 \\
(1.39 \pm 1.04 \text { per patient })^{*} \\
(66.0 \%)\end{array}$ \\
\hline $\begin{array}{l}\text { Patients with breast cancer } \\
\qquad(\mathrm{n}=8)\end{array}$ & 22 & $\begin{array}{c}12 \\
(54.5 \%)\end{array}$ \\
\hline $\begin{array}{l}\text { Patients with other cancers } \\
\qquad(\mathrm{n}=10)\end{array}$ & 16 & $\begin{array}{c}13 \\
(81.2 \%)\end{array}$ \\
\hline $\begin{array}{l}\text { Patients with fertility problems } \\
\qquad(\mathrm{n}=21)\end{array}$ & $65 / 74$ & $\begin{array}{c}52 \\
(2.48 \pm 1.83 \text { per patient })^{*} \\
(80.0 \%)\end{array}$ \\
\hline
\end{tabular}

* = statistically significant difference ( $P<0.05$; Mann-Whitney U test)

After ICSI, the fertilization and cleavage rates of 49 oocytes that matured in vitro were compared with 121 oocytes of the same patients that matured in vivo and were aspirated from their ovaries as mature oocytes (metaphase II [MII] oocyte meiosis) in the same cycle of controlled ovarian hormonal stimulation. Of the 121 oocytes obtained as mature oocytes, 69 oocytes were fertilized, representing a fertilization rate of $57.0 \%$. Sixty-one fertilized oocytes further developed into an embryo (Table 3). Fisher's exact test revealed no statistical differences in the proportions of fertilized oocytes, noncleaved zygotes, and embryos obtained by ICSI on in vitro and in vivo matured oocytes (Table 3 ). 
TABLE 3. Non-significant differences in fertilized oocytes, non-cleaved zygotes, and cleavage embryos obtained by intracytoplasmic sperm injection (ICSI) on in vitro matured and in vivo matured oocytes of patients with fertility problems (in the same cycles)

\begin{tabular}{ccc}
\hline $\begin{array}{c}\text { ICSI cycles } \\
(\mathbf{n}=17)\end{array}$ & $\begin{array}{c}\text { In vitro matured } \\
\text { oocytes }\end{array}$ & $\begin{array}{c}\text { In vivo matured } \\
\text { oocytes }\end{array}$ \\
\hline Number of microinjected oocytes & 49 & 121 \\
Fertilized oocytes & 27 & 69 \\
Non-cleaved zygotes & $(55.1 \%)$ & $(57.0 \%)$ \\
Cleavage embryos & 4 & 8 \\
& $(15.0 \%)$ & $(11.6 \%)$ \\
\hline
\end{tabular}

* = non-significant differences, as revealed by Fisher's exact test

TABLE 4. Non-significant differences in results of intracytoplasmic sperm injection (ICSI) cycles (fertilized oocytes, non-cleaved zygotes, cleavage embryos) on in vitro matured oocytes of patients with fertility problems regarding the number of immature (germinal vesicle [GV]) oocytes

\begin{tabular}{ccc}
\hline ICSI cycles $(\mathrm{n}=17)$ & $\leq 30 \%$ GV oocytes & $\geq 31 \%$ GV oocytes \\
\hline Female age (years) & $34.4 \pm 3.0$ & $34.3 \pm 5.6$ \\
Number of microinjected oocytes & 16 & 33 \\
Fertilized oocytes & $11(69.0 \%)$ & $16(48.5 \%)$ \\
Non-cleaved zygotes & $0(0 \%)$ & $4(25.0 \%)$ \\
Cleavage embryos & $11(100 \%)$ & $12(75 \%)$ \\
\hline
\end{tabular}

Non-significant differences, as revealed by Fisher's exact test

In patients with an increased proportion of immature $(\mathrm{GV})$ oocytes $(\geq 31 \%)$, there was a tendency for a lower proportion of fertilized oocytes and a higher proportion of non-cleaved zygotes, but the differences were not statistically significant (Table 4).

\section{Discussion}

The results of this research show that cancer and control healthy patients with fertility problems did not differ in the number and quality of oocytes after controlled hormonal stimulation of their ovaries, which is positive. In cancer patients, there was even a significantly lower proportion of immature oocytes than in patients with fertility problems. However, in patients with cancer, fewer oocytes per patient matured in vitro than in patients with fertility problems $(1.39 \pm 1.04$ vs. $2.48 \pm 1.83$, $\mathrm{P}<$ 0.05). Following ICSI of oocytes in patients with fertility problems, the fertilization and embryo cleavage rates were approximately the same in oocytes that matured in vitro and in vivo in the same patients, in the same cycles of controlled hormonal stimulation of the ovaries. This is also to be expected in cancer patients.

The proportion of mature, MII oocytes in the patients with fertility problems included in this research was relatively low (56.4\%) compared to the internationally accepted reference value of 70$80 \%{ }^{28}$, because we included mainly patients with a higher proportion of immature oocytes which did not reflect the average condition; in cancer patients, the proportion of mature oocytes was higher $(70 \%)$ and within the reference value. ${ }^{28}$ The number and quality of oocytes in cancer patients did not differ between different cancers and from control patients with fertility problems. The same has been found in other studies for different types of cancer such as breast cancer, lymphoma, gliomas and other cancers..$^{29,30}$ For breast cancer, the results of various studies are otherwise contradictory. In a study by Malacarne et al., as in our study, the average number of oocytes obtained per breast cancer patient after ovarian stimulation did not differ significantly from healthy control women including oocyte donors, women with fertility preservation for non-medical reasons, and female partners of infertile men in an IVF program ${ }^{31}$; it was concluded that patients with breast cancer undergoing controlled ovarian hormonal stimulation for fertility preservation can expect the ovarian response predicted for their age. The results obtained by different studies do not support the notion of a negative impact of the breast cancer gene 1/2 (BRCA1/2) mutation on the ovarian response of women with breast cancer. ${ }^{31-33}$ Nevertheless, the results of some other studies suggest the reduced number and maturity of oocytes obtained for cryostorage in patients with breast cancer ${ }^{34}$, which may be attributed to the higher grade of cancer ${ }^{35}$ or different expression of hormonal receptors. ${ }^{36}$

There is little data in the literature on how different cancers affect the oocyte IVM in cancer patients. The oocyte IVM rate in breast cancer patients was found to be approximately 53.2 to $64.2 \%$ in the 
study of Shalom Paz et al. ${ }^{37}$, which is very similar to our study $(54.5 \%)$, or slightly higher $-62.0 \%, 66.0 \%$ or $66.7 \%$ in some other studies. ${ }^{22,32}$ In this study, the proportion of immature oocytes matured in vitro tended to be lower in breast cancer patients $(54.5 \%)$ than in patients with fertility problems $(80.0 \%)$, while this was not observed in patients with other cancers $(81.2 \%)$. Although, the difference was not significant, possibly due to relatively low number of patients and oocytes included. In a study conducted by Liu et al., 811 genes were identified that were expressed differently in malignant breast tissue compared to healthy breast tissue ${ }^{38}$; among the up-regulated genes was also a group of genes involved in the cell cycle and progesteronemediated oocyte maturation. For cancer patients, Cohen et al. found that the mean oocyte maturation rate in stimulated IVF cycles was $38 \%{ }^{39}$, which is significantly lower than in our study $(66.0 \% ; 54.5 \%$ in breast cancer and $81.0 \%$ in other cancers). In our study, IVM of oocytes in coculture with cumulus cells from mature oocytes in the same patients may have been beneficial, at least in part providing an ovarian niche. ${ }^{26}$ Also Chatroudi et al. found that cumulus cell supplementation in IVM culture media enhances the viability of human embryos (blastocysts) after IVF. ${ }^{40}$ The rates of in vitro matured oocytes in cancer patients and patients with fertility problems in our study were very similar to the published rates of IVM of immature oocytes in the usual IVF program, where $65.0 \%, 68.7 \%, 68.9 \%$ and $69.7 \%$ maturation rates were obtained in different maturation media. ${ }^{41-43}$ Oktay et al. reported the $45 \%$ increase in mature oocyte yield after IVM of immature oocytes after controlled hormonal stimulation of the ovaries in breast cancer patients and a high fertilization rate of these oocytes. ${ }^{44}$ Moreover, an IVM of oocytes retrieved without hormonal stimulation of the ovaries was considered for fertility preservation in breast cancer patients to avoid the ovarian stimulation, shorten the time to oocyte retrieval, and not to increase both the serum estradiol level and delay in cancer treatment. ${ }^{45-47}$

It should be noted that our study was limited to a relatively small number of patients involved and a small number of oocytes. In cancer patients and patients with fertility problems, we tried to perform as comparable controlled hormonal stimulation of the ovaries as possible using a GnRH antagonist. Nevertheless, we also had to take certain safety precautions in cancer patients. In these patients, the ovarian hormonal stimulation was initiated immediately after they have been sent to our department, no matter of the cycle phase to be fast and prevent further progression of disease. The oocyte maturation in cancer patients was initiated by $\mathrm{GnRH}$ analogue to prevent hyperstimulation, but more patients with less than 10 follicles were also treated with Ovitrelle similar to patients with fertility problems. Thus, in most patients, oocyte maturation was triggered by Ovitrelle. If we used exactly the same method of hormonal ovarian stimulation and triggering oocyte maturation in cancer patients and patients with fertility problems, there might be more immature oocytes in cancer patients, but this was not possible for cancer-related safety reasons.

In addition, for safety reasons, breast cancer patients were also treated with an aromatase inhibitor, letrozole, to prevent an increase in estradiol and worsening of the disease. Thus, based on our own experience and literature ${ }^{48,49}$, we believe that both random start of hormonal stimulation in cancer patients and use of aromatase inhibitor in patients with breast cancer do not affect the number, maturity and in vitro maturation of oocytes obtained in these patients.

Letrozole treatment may also increase the intraovarian androgen levels, which have a negative impact on granulosa cells (apoptosis) in the late antral and pre-ovulatory follicles. ${ }^{50}$ In this research, granulosa (cumulus) cells were used in co-culture for oocyte maturation, which may lower the maturation rate. However, we performed in vitro maturation of oocytes with cumulus cells of mature oocytes because our previous work showed that coculture with cumulus cells does not affect the proportion of in vitro matured oocytes, but improves the molecular status of oocytes (gene expression profile) compared to oocytes matured in vivo. ${ }^{26}$

In patients with fertility problems, we determined the FSH, LH and AMH levels in early follicular phase of the cycle as well as the number of antral follicles. For cancer patients, we have no such data. Only informative ovarian scan with antral follicle estimation was performed at the beginning of ovarian stimulation.

In our study, approximately the same proportion of oocytes were fertilized and further cleaved into an embryo after ICSI of in vitro and in vivo matured oocytes. Some studies have shown poorer embryo development and live birth rates with in vitro matured oocytes ${ }^{51,52}$, which could be linked to structural and morphologic differences in human oocytes after IVM ${ }^{53}$ due to suboptimal maturation medium and lack of ovarian niche. It needs to be point out that in our study, oocyte IVM was performed in coculture with cumulus cells from ma- 
ture oocytes of the same patients thus providing a degree of ovarian niche. In spite of a lower rate of good-quality embryos and different developmental dynamics of embryos, pregnancy rates as well as live births did not necessarily differ after oocyte IVM, as found by Roesner et al. ${ }^{54}$ Moreover, in a matched setting between IVM and IVF babies born from women with polycystic ovaries, no significant increased risk associated with IVM has been identified in 2-year-old singletons born after IVM and after a mean follow-up up to 7.5 years. ${ }^{55,56}$ In general, more studies are urgently required to improve IVM -vitrification method to successfully preserve oocytes collected from cancer patients. ${ }^{57,58}$

\section{Conclusions}

We may conclude that 'rescue' of immature oocytes with IVM is a useful strategy to improve the mature oocyte yield of fertility preservation cycles in cancer patients. Immature oocytes retrieved during oocyte and also embryo cryopreservation cycles in cancer patients should not be discarded in order to improve the future potential of pregnancy in these patients. Their immature oocytes can mature in vitro comparable to healthy controls. After ICSI, approximately the same proportion of in vitro matured oocytes could be fertilized and developed into an embryo as in oocytes matured in vivo.

\section{Acknowledgments}

We would like to thank all colleagues, gynecologists, embryologists, and nurses at the Department of Gynecology and Obstetrics, Reproductive Unit (IVF), who in any way helped in this work. We also thank all the patients who kindly donated immature oocytes for this research. Last but not least, we would also like to thank our institution, University Medical Centre Ljubljana, which funded this research as part of a tertiary research project.

\section{References}

1. Yeo W, Pang E, Liem GS, Suen JJS, Ng RYW, Yip CCH, et al. Menopausal symptoms in relationship to breast cancer-specific quality of life after adjuvant cytotoxic treatment in young breast cancer survivors. Health Qual Life Outcomes 2020; 18: 24. doi: 10.1186/s12955-020-1283-x

2. Silvestris $E$, Dellino $M$, Cafforio $P$, Paradiso AV, Cormio G, D'Oronzo S. Breast cancer: an update on treatment-related infertility. J Cancer Res Clin Oncol 2020; 146: 647-57. doi: 10.1007/s00432-020-03136-7
3. Felicetti F, Castiglione A, Biasin E, Fortunati N, Dionisi-Vici M, Matarazzo P, et al. Effects of treatments on gonadal function in long-term survivors of pediatric hematologic malignancies: a cohort study. Pediatr Blood Cancer 2020; 67: e28709. doi: 10.1002/pbc.28709

4. Yeganeh L, Boyle JA, Gibson-Helm M, Teede H, Vincent AJ. Women's perspectives of early menopause: development of a word cloud. Climacteric 2020; 23: 417-20. doi: 10.1080/13697137.2020.1730318

5. Delattre S, Segers I, Van Moer E, Drakopoulos P, Mateizel I, Enghels L, et al. Combining fertility preservation procedures to spread the eggs across different baskets: a feasibility study. Hum Reprod 2020; 35: 2524-36. doi: 10.1093/humrep/deaa193.

6. Dinas KD. Fertility counseling and preservation for breast cancer patients Adv Exp Med Biol 2020; 1252: 181-87. doi: 10.1007/978-3-030-41596-9_25

7. Bénard J, Duros S, El Hachem H, Sonigo C, Sifer C, Grynberg M. Freezing oocytes or embryos after controlled ovarian hyperstimulation in cancer patients: the state of the art. Future Oncol 2016; 12: 1731-41. doi: 10.2217/ fon-2016-0095

8. de Pedro M, Otero B, Martín B. Fertility preservation and breast cancer: a review. Ecancer 2015; 9: 503. doi: 10.3332/ecancer.2015.503

9. Kasum M, Beketić-Orešković L, Peddi PF, Orešković S, Johnson RH. Fertility after breast cancer treatment. Eur J Obstet Gynecol Reprod Biol 2014; 173: 13-8. doi: 10.1016/j.ejogrb.2013.11.009

10. Peccatori FA, Pup LD, Salvagno F, Guido M, Sarno MA, Revelli A, et al. Fertility preservation methods in breast cancer. Breast Care 2012; 7: 197202. doi: $10.1159 / 000339671$

11. Redig AJ, Brannigan R, Stryker SJ, Woodruff TK, Jeruss JS. Incorporating fertility preservation into the care of young oncology patients. Cancer 2011; 117: 4-10. doi: 10.1002/cncr.25398

12. Meirow D. Reproduction post-chemotherapy in young cancer patients. Mol Cell Endocrinol 2000; 169: 123-31. doi: 10.1016/s0303-7207(00)00365-8

13. Specchia C, Baggiani A, Immediata V, Ronchetti C, Cesana A, Smeraldi A, et al. Oocyte cryopreservation in oncological patients: eighteen years' experience of a tertiary care referral center. Front Endocrinol 2019; 10: 600. doi: 10.3389/fendo.2019.00600

14. Gook DA, Edgar DH. Cryopreservation of female reproductive potential. Best Pract Res Clin Obstet Gynaecol 2019; 55: 23-36. doi: 10.1016/j.bpobgyn.2018.08.005

15. Cobo A, García-Velasco J, Domingo J, Pellicer A, Remohí J. Elective and oncofertility preservation: factors related to IVF outcomes. Hum Reprod 2018; 33: 2222-31. doi: 10.1093/humrep/dey321

16. Dolmans MM, Donnez J. Fertility preservation in women for medical and social reasons: oocytes vs ovarian tissue. Best Pract Res Clin Obstet Gynaecol 2020; 21: S1521-6934(20)30122-X. doi: 10.1016/j.bpobgyn.2020.06.011

17. Khalili MA, Shahedi A, Ashourzadeh S, Nottola SA, Macchiarelli G, Palmerini MG. Vitrification of human immature oocytes before and after in vitro maturation: a review. J Assist Reprod Genet 2017; 34: 1413-26. doi: 10.1007/ s10815-017-1005-4

18. Ata B, Chian RC, Tan SL. Cryopreservation of oocytes and embryos for fertility preservation for female cancer patients. Best Pract Res Clin Obstet Gynaecol 2010; 24: 101-12. doi: 10.1016/j.bpobgyn.2009.11.007

19. El Hachem H, Atallah D, Grynberg M. Fertility preservation in breast cancer patients. Future Oncol 2014; 10: 1767-77. doi: 10.2217/fon.14.55

20. Muñoz E, Domingo J, De Castro G, Lorenzo I, García-Velasco JA, Bellver J, et al. Ovarian stimulation for oocyte vitrification does not modify diseasefree survival and overall survival rates in patients with early breast cancer. Reprod Biomed Online 2019; 39: 860-67. doi: 10.1016/j.rbmo.2019.07.003

21. Rodgers RJ. Fertility preservation in breast cancer patients. Minerva Ginecol 2019; 71: 196-206. doi: 10.23736/S0026-4784.19.04409-5

22. Grynberg M, Poulain M, le Parco S, Sifer C, Fanchin R, Frydman N. Similar in vitro maturation rates of oocytes retrieved during the follicular or luteal phase offer flexible options for urgent fertility preservation in breast cancer patients. Hum Reprod 2016; 31: 623-9. doi: 10.1093/humrep/dev325

23. Grynberg M, Mayeur Le Bras A, Hesters L, Gallot V, Frydman N. First birth achieved after fertility preservation using vitrification of in vitro matured oocytes in a woman with breast cancer. Ann Oncol 2020; 31: 541-42. doi: 10.1016/j.annonc.2020.01.005 
24. World Health Organisation. WHO laboratory manual for the examination and processing of human semen. Geneva: WHO Press; 2010.

25. Stimpfel M, Vrtacnik-Bokal E, Virant-Klun I. No difference in mitochondrial distribution is observed in human oocytes after cryopreservation. Arch Gynecol Obstet 2017; 296: 373-81. doi: 10.1007/s00404-017-4428-3

26. Virant-Klun I, Bauer C, Ståhlberg A, Kubista M, Skutella T. Human oocyte maturation in vitro is improved by co-culture with cumulus cells from mature oocytes. Reprod Biomed Online 2018; 36: 508-23. doi: 10.1016/j. rbmo.2018.01.011

27. Stimpfel M, Verdenik I, Zorn B, Virant-Klun I. Magnetic-activated cell sorting of non-apoptotic spermatozoa improves the quality of embryos according to female age: a prospective sibling oocyte study. J Assist Reprod Genet 2018; 35: 1665-74. doi:10.1007/s10815-018-1242-1

28. ESHRE Special Interest Group of Embryology and Alpha Scientists in Reproductive Medicine. The Vienna consensus: report of an expert meeting on the development of art laboratory performance indicators. Hum Reprod Open 2017; 2017: 1-17. doi: 10.1093/hropen/hox011

29. Lefebvre $T$, Mirallié $S$, Leperlier $F$, Reignier A, Barrière $P$, Fréour T. Ovarian reserve and response to stimulation in women undergoing fertility preservation according to malignancy type. Reprod Biomed Online 2018; 37: 201-7. doi: 10.1016/j.rbmo.2018.04.047

30. Nordan T, Thomas AM, Ginsburg ES, Wen PY, Dolinko AV, Bortoletto P. Fertility preservation outcomes in women with gliomas: a retrospective case-control study. J Neurooncol 2020; 147: 371-76. doi: 10.1007/s11060020-03429-4

31. Malacarne E, Devesa M, Martinez F, Rodriguez I, Coroleu B. COH outcomes in breast cancer patients for fertility preservation: a comparison with the expected response by age. J Assist Reprod Genet 2020; 37: 3069-76. doi: $10.1007 / \mathrm{s} 10815-020-01944-\mathrm{x}$

32. Grynberg M, Dagher Hayeck B, Papanikolaou EG, Sifer C, Sermondade N, Sonigo C. BRCA1/2 gene mutations do not affect the capacity of oocytes from breast cancer candidates for fertility preservation to mature in vitro. Hum Reprod 2019; 34: 374-79. doi: 10.1093/humrep/dey358

33. Gunnala V, Fields J, Irani M, D'Angelo D, Xu K, Schattman G, et al. BRCA carriers have similar reproductive potential at baseline to noncarriers: comparisons in cancer and cancer-free cohorts undergoing fertility preservation. Fertil Steril 2019; 111: 363-71. doi: 10.1016/j.fertnstert.2018.10.014

34. Moria A, Das M, Shehata F, Holzer H, Son WY, Tulandi T. Ovarian reserve and oocyte maturity in women with malignancy undergoing in vitro maturation treatment. Fertil Steril 2011; 95: 1621-3. doi: 10.1016/j.fertnstert.2010.12.041

35. Volodarsky-Perel A, Cai E, Tulandi T, Son WY, Suarthana E, Buckett W. Influence of stage and grade of breast cancer on fertility preservation outcome in reproductive-aged women. Reprod Biomed Online 2020; 40: 215-22. doi: 10.1016/j.rbmo.2019.11.006

36. Balayla J, Tulandi T, Buckett W, Holzer H, Steiner N, Shrem G, et al. Outcomes of ovarian stimulation and fertility preservation in breast cancer patients with different hormonal receptor profiles. J Assist Reprod Genet 2020; 37: 913-21. doi: 10.1007/s10815-020-01730-9

37. Shalom-Paz E, Almog B, Shehata F, Huang J, Holzer $H$, Chian RC, et al. Fertility preservation for breast-cancer patients using IVM followed by oocyte or embryo vitrification. Reprod Biomed Online 2010; 21: 566-71. doi: 10.1016/j.rbmo.2010.05.003

38. Liu Z, Liang G, Tan L, Su AN, Jiang W, Gong C. High-efficient screening method for identification of key genes in breast cancer through microarray and bioinformatics. Anticancer Res 2017; 37: 4329-35. doi: 10.21873/ anticanres.11826

39. Cohen Y, Tannus S, Volodarsky-Perel A, Son WY, Tulandi T, Buckett W. Added benefit of immature oocyte maturation for fertility preservation in women with malignancy. Reprod Sci 2020; 27: 2257-64. doi: 10.1007/s43032-02000245-z

40. Chatroudi MH, Khalili MA, Ashourzadeh S, Anbari F, Shahedi A, Safari S. Growth differentiation factor 9 and cumulus cell supplementation in in vitro maturation culture media enhances the viability of human blastocysts. Clin Exp Reprod Med 2019; 46: 166-72. doi: 10.5653/cerm.2019.00402
41. Sacha CR, Kaser DJ, Farland LV, Srouji S, Missmer SA, Racowsky C. The effect of short-term exposure of cumulus-oocyte complexes to in vitro maturation medium on yield of mature oocytes and usable embryos in stimulated cycles. J Assist Reprod Genet 2018; 35: 841-49. doi: 10.1007/ s10815-018-1155-z

42. Pongsuthirak $P$, Songveeratham S, Vutyavanich T. Comparison of blastocyst and Sage media for in vitro maturation of human immature oocytes. Reprod Sci 2015; 22: 343-6. doi: 10.1177/1933719114542027

43. Junk SM, Yeap D. Improved implantation and ongoing pregnancy rates after single-embryo transfer with an optimized protocol for in vitro oocyte maturation in women with polycystic ovaries and polycystic ovary syndrome. Fertil Steril 2012; 98: 888-92. doi: 10.1016/j.fertnstert.2012.06.055

44. Oktay K, Buyuk E, Rodriguez-Wallberg KA, Sahin G. In vitro maturation improves oocyte or embryo cryopreservation outcome in breast cancer patients undergoing ovarian stimulation for fertility preservation. Reprod Biomed Online 2010; 20: 634-8. doi: 10.1016/j.rbmo.2010.01.012

45. D'Hondt C, Vanhoeij M, Van Moer E, Segers I, Fontaine C, Tournaye H, et al. Fertility preservation does not delay the initiation of chemotherapy in breast cancer patients treated with adjuvant or neo-adjuvant chemotherapy. Breast Cancer Res Treat 2020; 184: 433-44. doi: 10.1007/s10549020-05858-1

46. Huang JY, Chian RC, Gilbert L, Fleiszer D, Holzer H, Dermitas E, et al. Retrieval of immature oocytes from unstimulated ovaries followed by in vitro maturation and vitrification: A novel strategy of fertility preservation for breast cancer patients. Am J Surg 2010; 200: 177-83. doi: 10.1016/j. amjsurg.2009.04.004

47. Oktay K, Demirtas E, Son WY, Lostritto K, Chian RC, Tan SL. In vitro maturation of germinal vesicle oocytes recovered after premature luteinizing hormone surge: description of a novel approach to fertility preservation. Fertil Steril 2008; 89: 228.e19-22. doi: 10.1016/j.fertnstert.2007.02.028

48. Marklund A, Eloranta S, Wikander I, Kitlinski ML, Lood M, Nedstrand E, et al. Efficacy and safety of controlled ovarian stimulation using $\mathrm{GnRH}$ antagonist protocols for emergency fertility preservation in young women with breast cancer-a prospective nationwide Swedish multicenter study. Hum Reprod 2020; 35: 929-38. doi: 10.1093/humrep/deaa029

49. American Society for Reproductive Medicine. Fertility preservation in patients undergoing gonadotoxic therapy or gonadectomy: a committee opinion. Practice Committee of the American Society for Reproductive Medicine. Fertil Steril 2019; 112: 1022-33. doi: 10.1016/j.fertnstert.2019.09.013

50. Rose $\mathrm{BI}, \mathrm{Brown} \mathrm{SE}$. A review of the physiology behind letrozole applications in infertility: are current protocols optimal? J Assist Reprod Genet 2020; 37: 2093-104. doi: 10.1007/s10815-020-01892-6

51. Santiquet NW, Greene AF, Becker J, Barfield JP, Schoolcraft WB, Krisher RL. A pre-in vitro maturation medium containing cumulus oocyte complex ligand-receptor signaling molecules maintains meiotic arrest, supports the cumulus oocyte complex and improves oocyte developmental competence. Mol Hum Reprod 2017; 23: 594-606. doi: 10.1093/molehr/gax032

52. Walls ML, Hunter T, Ryan JP, Keelan JA, Nathan E, Hart RJ. In vitro maturation as an alternative to standard in vitro fertilization for patients diagnosed with polycystic ovaries: a comparative analysis of fresh, frozen and cumulative cycle outcomes. Hum Reprod 2015; 30: 88-96. doi: 10.1093/humrep/deu248

53. Walls ML, Hart R, Keelan JA, Ryan JP. Structural and morphologic differences in human oocytes after in vitro maturation compared with standard in vitro fertilization. Fertil Steril 2016; 106: 1392-98. doi: 10.1016/j.fertnstert.2016.08.014

54. Roesner S, Dietrich JE, Weigert J, Montag M, Toth B, Strowitzki T. Time-lapse imaging reveals differences in growth dynamics of embryos after in vitro maturation compared with conventional stimulation. Fertil Steril 2017; 107: 606-12. doi: 10.1016/j.fertnstert.2016.12.026

55. Belva F, Roelants M, Vermaning S, Desmyttere S, De Schepper J, Bonduelle $\mathrm{M}$, et al. Growth and other health outcomes of 2-year-old singletons born after IVM versus controlled ovarian stimulation in mothers with polycystic ovary syndrome. Hum Reprod Open 2020; 2020: hoz043. doi: 10.1093/ hropen/hoz043

56. Yu EJ, Yoon TK, Lee WS, Park EA, Heo JY, Ko YK, et al. Obstetrical, neonatal, and long-term outcomes of children conceived from in vitro matured oocytes. Fertil Steril 2019; 112: 691-99. doi: 10.1016/j.fertnstert.2019.05.034 
57. Son WY, Henderson S, Cohen Y, Dahan M, Buckett W. Immature Oocyte for Fertility Preservation. Front Endocrinol 2019; 10: 464. doi: 10.3389/ fendo.2019.00464

58. Shirasawa $H$, Terada $Y$. In vitro maturation of human immature oocytes for fertility preservation and research material. Reprod Med Biol 2017; 16: 25867. doi: $10.1002 / \mathrm{rmb} 2.12042$ 